\title{
Proliferation and tissue remodeling in cancer: the hallmarks revisited
}

\author{
EK Markert ${ }^{1}$, AJ Levine ${ }^{1,2,3}$ and A Vazquez ${ }^{*, 1,2,4}$
}

\begin{abstract}
Although cancers are highly heterogeneous at the genomic level, they can manifest common patterns of gene expression. Here, we use gene expression signatures to interrogate two major processes in cancer, proliferation and tissue remodeling. We demonstrate that proliferation and remodeling signatures are partially independent and result in four distinctive cancer subtypes. Cancers with the proliferation signature are characterized by signatures of p53 and PTEN inactivation and concomitant Myc activation. In contrast, remodeling correlates with RAS, HIF-1 $\alpha$ and NF $\kappa$ B activation. From the metabolic point of view, proliferation is associated with upregulation of glycolysis and serine/glycine metabolism, whereas remodeling is characterized by a downregulation of oxidative phosphorylation. Notably, the proliferation signature correlates with poor outcome in lung, prostate, breast and brain cancer, whereas remodeling increases mortality rates in colorectal and ovarian cancer.
\end{abstract}

Cell Death and Disease (2012) 3, e397; doi:10.1038/cddis.2012.140; published online 4 October 2012

Subject Category: Cancer

The human body is organized into a hierarchy of level structures (cells, organs and organism) with their hierarchically interrelated functions. In turn, cancer can be organized into its level structures as well: the cancer cell, the primary tumor and the systemic disease. On each level, cancer deregulates the normal functions and activities associated with that level's structure. At the lowest level we have the cell, and among its main functions controlled cell renewal and proliferation. In turn, deregulation of cell proliferation is a prerequisite for carcinogenesis. ${ }^{1}$ One level up, cells are arranged into tissue, which provides structural support and organization, nutrient supplies via blood vessels, and inflammation and wound-healing activities in response to tissue damage or nutrient stress. ${ }^{1}$ To form a primary tumor, the cancer cells should master the remodeling of the cancerous and adjacent tissue, attracting blood vessels and transforming the surrounding tissue, to promote tumor growth and local invasion. Finally at the organism level, cancer affects global functions of the organism, evading its immune defenses, utilizing the organism's blood circuitry to spread and metastasize, and inducing systemic metabolic alteration such as cachexia. ${ }^{1}$ It is this final systemic state of the disease that ultimately results in patient death.

This line of reasoning suggests that a systematic classification of cancer could display the same hierarchical organization. Deregulated proliferation has been studied in great detail and we now know a great deal about the signaling pathway alterations that allow cancer cells to master this major phenotype at the cellular level. ${ }^{2,3}$ Tissue alterations, such as angiogenesis, hypoxia and inflammation, have also been significantly investigated and the signaling pathway alterations driving these tissue phenotypes discovered. ${ }^{4-6}$ Systemic alterations such as metastasis and cachexia have been investigated as well, ${ }^{7,8}$ albeit to a lesser extent due to experimental challenges. These studies have, over time, been performed in many cancers, thus generating the base for a more universal, across-cancers analysis. However, most of the research has focused on the particularities of individual cancer types that result from the overwhelming number of genomic alterations that can drive these phenotypes. ${ }^{9-11}$

In this paper, we follow a different approach, focusing on the commonalities among all cancers rather than on their particularities. To this end, we analyze a large collection of gene expression data from various tumor sites. We show how cell- and tissue-level aspects comprise two main axes of cancer development, proliferation $(P)$ and tissue remodeling (R). We leave aside the third systemic level of organization because of the present lack of appropriate experimental data needed for its characterization. We show how tumors across multiple tissues can be characterized by their activities of proliferation and remodeling. We furthermore correlate these key phenotypes with a variety of the most common pathway alterations in cancer. In this context, we also include major metabolic pathways and transcriptional patterns reflecting loss of lineage or differentiation control, manifested as an embryonic stem cell (ESC-like phenotype ${ }^{12-14}$ ) or a mesenchymal phenotype. ${ }^{15-17}$ Finally, we analyze survival data across

\footnotetext{
${ }^{1}$ The Simons Center for Systems Biology, Institute for Advanced Study, Princeton, NJ, USA; ${ }^{2}$ The Cancer Institute of New Jersey, University of Medicine and Dentistry of New Jersey, Robert Wood Johnson Medical School, New Brunswick, NJ, USA; ${ }^{3}$ Department of Pediatrics, University of Medicine and Dentistry of New Jersey, Robert Wood Johnson Medical School, New Brunswick, NJ, USA and ${ }^{4}$ Department of Radiation Oncology and Center for Systems Biology, University of Medicine and Dentistry of New Jersey, Robert Wood Johnson Medical School, New Brunswick, NJ, USA

${ }^{*}$ Corresponding author: A Vazquez, The Cancer Institute of New Jersey, University of Medicine and Dentistry of New Jersey, Robert Wood Johnson Medical School, 195 Little Albany St., New Brunswick, NJ 08903, USA. Tel: +732 235 8918; Fax: +732 235 8869; E-mail: vazqueal@ umdnj.edu

Keywords: cancer pathways; cancer metabolism; cancer stem cell; meta-pathway; hallmarks

Abbreviations: P, proliferation; R, remodeling; EMT, epithelial mesenchymal transition; OxPhos, oxidative phosphorylation; SOG, serine biosynthesis, one carbon metabolism and the glycine cleavage system; ESC, embryonic stem cell; GSEA, gene set enrichment analysis

Received 17.7.12; accepted 20.8.12; Edited by G Raschella'
} 
cancers, to assess the impact of these two fundamental processes as a function of site.

\section{Results}

Gene expression signatures for proliferation and remodeling. Proliferation and remodeling require the expression of specific pathways. In turn, the consensus expression of genes in these pathways can be used as a surrogate to quantify the degree of proliferation and remodeling based on gene expression profiles. Gene expression signature analysis has been shown to accurately reflect major cellular and tissue phenotypes and to provide a robust tool for a systematic analysis across data sets. In the case of proliferation these pathways include well-known processes associated with cell cycle progression and cell division: the $\mathrm{G}_{1} / \mathrm{S}$ transition, DNA replication, telomere organization, chromosome segregation, DNA packaging, the $\mathrm{G}_{2} / \mathrm{M}$ transition and cell division. In the category of remodeling, we envision processes involving an interaction with the microenvironment or a change relative to the microenvironment. Here, we include cell junction organization, cell adhesion, cell migration, angiogenesis, cytokine production, inflammation and response to wounding. These are not by any means exhaustive lists, but a representative selection of processes that are involved in proliferation and the interaction between the cell and its microenvironment. To quantify the manifestation of these different processes, we define gene signatures based on the gene ontology annotations or literature reports, enumerating the genes implicated in these functions according to our current knowledge (Materials and Methods).

We tested the resulting proliferation and remodeling sub-signatures using reported gene expression data sets measured in experimental settings where the desired process was activated/inactivated. In an experiment where proliferation was induced by IGF-I (Figure 1a), the proliferation signatures were indeed present in all samples stimulated with IGF-I as opposed to the controls, while the tissue remodeling signatures were overall not significantly associated with control or case samples. In order to test the remodeling signatures, we chose an experimental setting where the epithelial mesenchymal transition (EMT) was induced by TGF $\beta$ stimulus (Figure $1 \mathrm{~b}$ ). We observe a clear transition from blue to red in all tissue remodeling signatures starting from the 8-h time point. We also note a decrease in the proliferation activity going hand in hand with the onset of tissue remodeling, suggesting that cells undergoing EMT reduce their proliferation activity while making the transition into mesenchymal type. We collected all genes in the subsignatures associated with proliferation and the sub-signatures associated with remodeling, to create the signatures of proliferation and remodeling, respectively. The proliferation and remodeling signatures capture the consensus expression of the respective sub-signatures in the controlled experiments (Figure 1).

We next investigated the correlation between the sub-signatures for the specific cellular processes (e.g., DNA replication or cell migration) and the signatures for proliferation and remodeling across 3931 samples from different cancers, including lung, prostate, breast, colorectal, brain and ovarian. The sub-signatures of proliferation are highly correlated with the proliferation signature, and the same was the case with the remodeling signatures (Table 1). However, all proliferation sub-signatures display a negative correlation with the remodeling signature, and all remodeling sub-signatures are negatively correlated with the proliferation signature (Table 1). This corroborates the observations in controlled experiments (Figures $1 \mathrm{a}$ and $\mathrm{b}$ ) in the context of human cancers. Notably, the negative correlations between the proliferation and the remodeling groups are low (bordering or lacking statistical significance) compared with the very strong correlations within each group of signatures. Together these data imply that the two meta-pathways proliferation and remodeling measure well-defined and clearly distinguished phenotypes; the weak-negative correlation suggests that these programs are observed in a statistically nearly independent fashion, with a subtle trend toward observation of opposing regimes. This is confirmed and further illustrated below using a classifying strategy.

Signaling pathways driving proliferation and remodeling. The achievement of the proliferation and remodeling
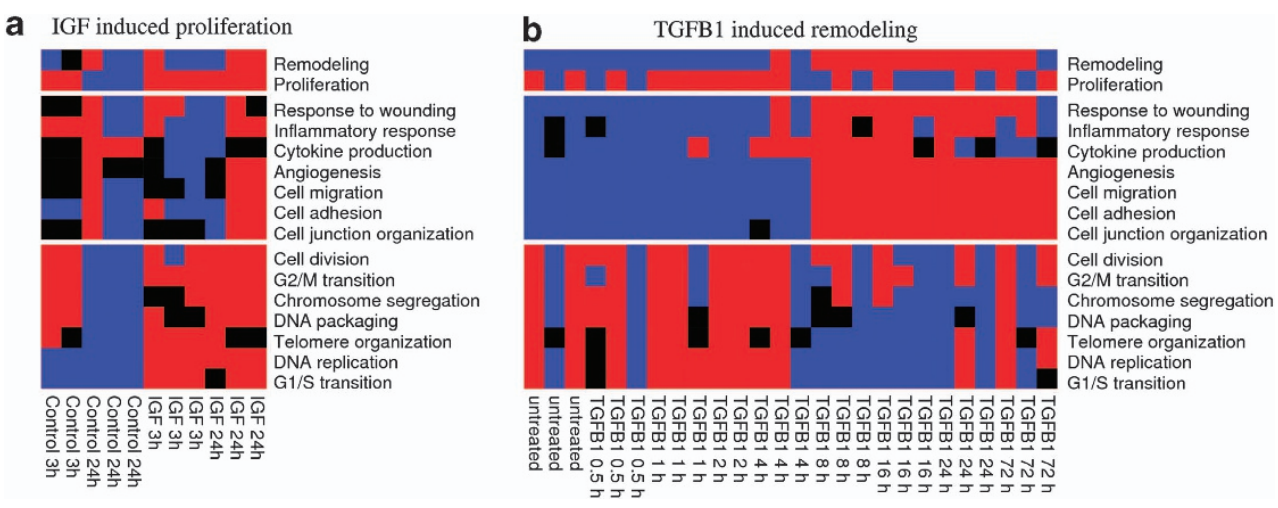

Figure 1 Proliferation and remodeling sub-signatures in controlled experiments. Heatmaps showing the proliferation and remodeling signatures in control experiments. The red indicates a significant upregulation, black no association, and blue a significant downregulation of the signature indicated on the right. (a) Induction of cell proliferation with IGF-I in MCF7 breast cancer cells, based on data from Creighton et al. ${ }^{48}$ (b) Induction of the EMT with TGF- $\beta$ in A549 human lung adenocarcinoma cells, based on data from Sartor et al. ${ }^{49}$ 
Table 1 Correlation between gene signatures

\begin{tabular}{lcc}
\hline Signature & Proliferation & Remodeling \\
\hline Proliferation sub-signatures & & \\
G $/$ S transition & 0.85 & -0.06 \\
DNA replication & 0.87 & -0.12 \\
Telomere organization & 0.75 & -0.14 \\
DNA packaging & 0.80 & -0.17 \\
Chromosome segregation & 0.81 & -0.14 \\
G2/M transition & 0.80 & -0.14 \\
Cell division & 0.87 & -0.06 \\
& & \\
Remodeling sub-signatures & & \\
Cell junction organization & -0.04 & 0.66 \\
Cell adhesion & -0.15 & 0.81 \\
Cell migration & -0.05 & 0.86 \\
Angiogenesis & -0.04 & 0.62 \\
Cytokine production & -0.02 & 0.67 \\
Inflammatory response & -0.07 & 0.82 \\
Response to wounding & -0.05 & \\
Signaling pathways & & -0.12 \\
Myc activation & & 0.06 \\
p53 inactivation & 0.68 & -0.11 \\
PTEN inactivation & 0.55 & 0.48 \\
HIF1alpha targets & 0.82 & 0.53 \\
RAS activation & 0.20 & 0.53 \\
NFKB activation & 0.10 & \\
Metabolism & 0.03 & 0.13 \\
Glycolysis & & -0.17 \\
Oxidative phosphorylation & 0.36 \\
SOG pathway & 0.47 & \\
Stem cell & & \\
Embryonic stem cell & 0.80 & \\
Mesenchyme development & -0.11 & \\
\hline
\end{tabular}

The Pearson correlation coefficient between the signatures on the first column and the signatures of proliferation and remodeling

phenotypes follows from the activation/ inactivation of signaling pathways regulating these processes. In the context of cancer this occurs owing to both the activation of oncogenes and the inactivation of tumor suppressors regulating proliferation and remodeling. To this end, we analyze gene signatures quantifying the activation of Myc, Ras, NF $\kappa \mathrm{B}$ and $\mathrm{HIF}-1 \alpha$ and inactivation of $\mathrm{p} 53$ and PTEN signaling pathways. Although this list is certainly not complete, it covers some of the most commonly altered pathways in human cancers.

We investigated the correlation between the signatures for activation/inactivation of signaling pathways and the signatures for proliferation and remodeling across the 3931 tumor samples (Table 1). We observe a strong positive correlation between Myc signaling activation, p53 signaling inactivation and PTEN signaling inactivation and the proliferation signature. This observation agrees with the role of Myc as a major promoter of proliferation and p53 and PTEN as major negative regulators of proliferation. In contrast, the Ras, NF $\kappa$ B and HIF-1 $\alpha$ signaling activation signatures are strongly correlated with the remodeling signatures. This observation was also expected, given the role of $\mathrm{NF} \kappa \mathrm{B}$ in inflammation and of $\mathrm{HIF}-1 \alpha$ in response to hypoxia. Ras activation has been commonly associated with increased proliferation. ${ }^{18}$ More recently, it has been shown that Kras oncogenic mutations activate glycosylation, ${ }^{19}$ which is known to have a key role in invasion and angiogenesis. ${ }^{20}$ Our observation does not exclude that Ras activation promotes proliferation under certain conditions, but rather suggests regulation of remodeling as its primary role.

Metabolic pathways fueling proliferation and remodeling. Proliferation and remodeling are both fueled by cell metabolism. Proliferating cells need energy and precursor metabolites to support the biosynthetic processes required to produce a new cell. ${ }^{21}$ Similarly, remodeling requires energy and precursor metabolites to support processes such as cell migration and secretion. For example, quiescent fibroblasts have high metabolic activity directed to protein synthesis. ${ }^{22}$ These requirements may be satisfied in different microenvironment conditions affecting nutrient availability, for example, hypoxic conditions. To investigate the metabolic requirements of proliferation and remodeling across tumors, we focus on signatures for energy metabolism. Glycolysis and oxidative phosphorylation (OxPhos) are the main recognized pathways for energy generation. Recently, we have discovered a third candidate pathway for ATP production involving serine biosynthesis, one carbon metabolism and the glycine cleavage system (SOG pathway). ${ }^{23}$ Elements of this pathway have been found upregulated in breast tumors ${ }^{24,25}$ and melanoma $^{26}$ and it has been shown to be essential for their development. Furthermore, most of the genes in this pathway are activated by Myc. ${ }^{23}$

We observe that the signatures of glycolysis, OxPhos and the SOG pathway are highly correlated with proliferation (Table 1), suggesting that tumors with a high proliferation signature are metabolically more active. The same conclusion was obtained when analyzing a metabolic model accounting for the metabolic demands of proliferating cells. ${ }^{23}$ This model predicts that the rate of glycolysis and the SOG pathway increase with increasing the proliferation rate. In contrast, OxPhos is predicted to first increase but then decrease with increasing the proliferation rate. ${ }^{23}$ The positive correlation between the signatures for OxPhos and proliferation can still be explained by the fact that the OxPhos signature is negatively correlated with the remodeling signature, which is in turn negatively correlated with the proliferation signature (Table 1).

Proliferation and remodeling define different stem cell phenotypes. The definition of cancer stem cells capable of recapitulating tumor development is quite challenging. The EMT can generate cells with stem cell properties, such as the CD44 + /CD24 - antigen phenotype. ${ }^{15,27}$ In an independent line of research, it has been shown that subsets of breast, liver and prostate cancers manifest signatures characteristic of ESCs. ${ }^{14,28,29}$ To investigate the relationship between these stem-cell like phenotypes and proliferation/remodeling, we studied gene signatures for ESC and mesenchymal development. We observe that the ESC signature is highly correlated with the proliferation signatures, whereas the mesenchyme development signature is highly correlated with the remodeling signatures (Table 1). The association between the ESC signature and proliferation has been previously noticed. ${ }^{12}$ In fact, ESCs undergo very fast proliferation during the initial steps of development. ${ }^{30,31}$ The association between mesenchymal development 
a

Signature expression in cancers by $\mathrm{P} / \mathrm{R}$ group

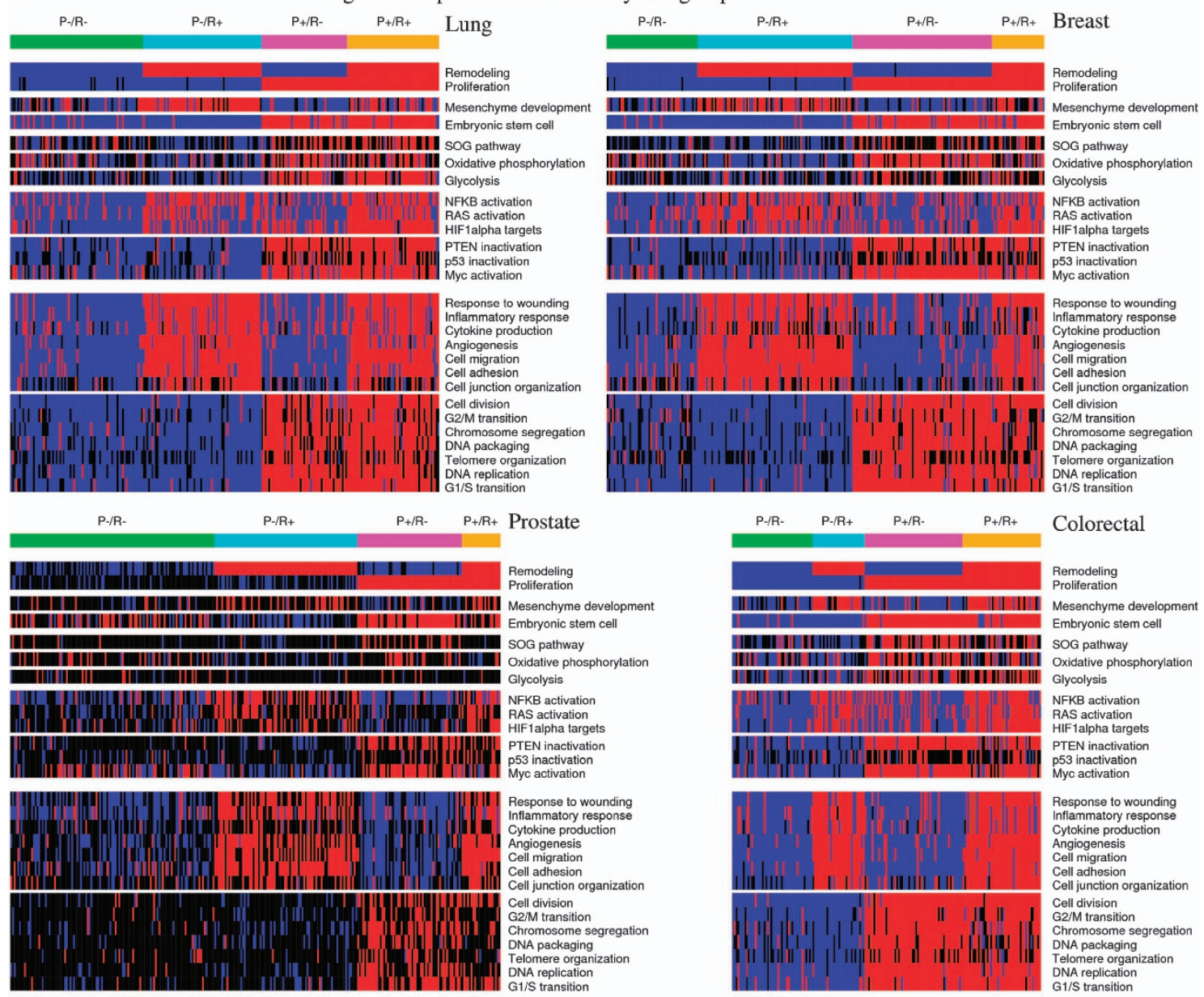

b

Signature enrichment in $\mathrm{P} / \mathrm{R}$ groups across cancers

$P-1 R_{+}$

$\mathrm{P}+/ \mathrm{R}$

$\mathrm{P}+/ \mathrm{R}+$

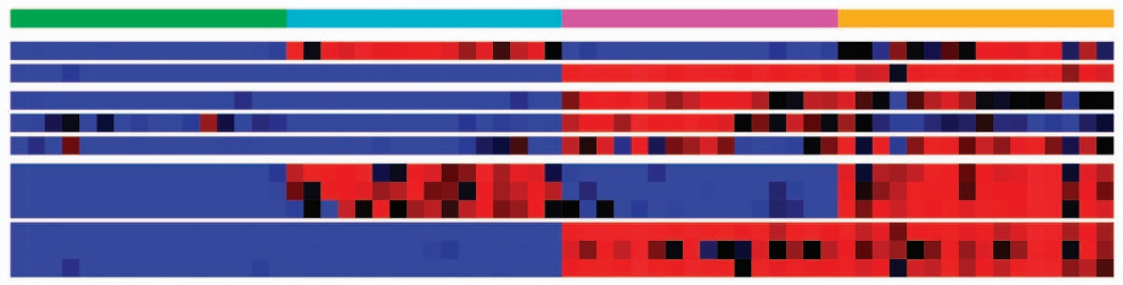

Mesenchyme development Embryonic stem cell SOG pathway Oxidative phosphorylatio Glycolysis NFKB activation
RAS activation RAS activation
HIF1alpha targets PTEN inactivation p53 inactivation

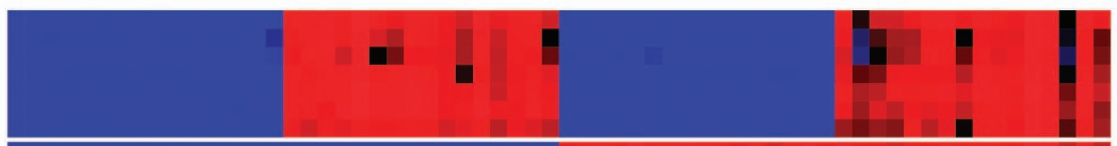
Response to wounding
Inflammatory response Inflammatory respons
Cytokine production Angiogenesis Cell migration Cell junction organization

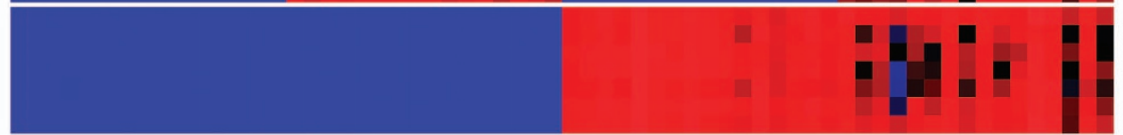
Cell division Chromosome segregation DNA packaging Telomere organization

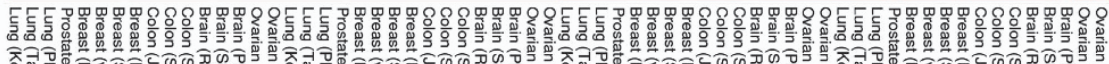
G1/S transition

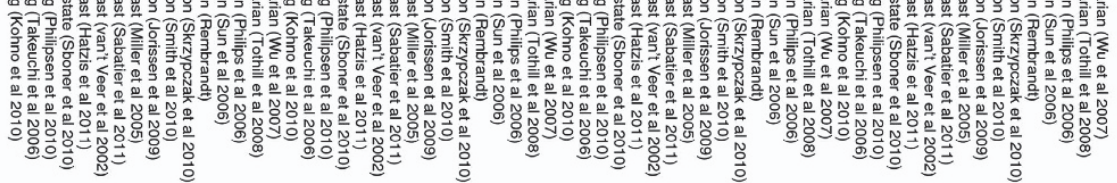

Figure 2 Gene expression signatures by P/R subtype. (a) Gene signatures expression in tumor samples from different cancer types. (b) Overall gene signature enrichment across cancer data sets by P/R subtype. In this heat map, a column represents the subset of tumors in the data set indicated in the bottom that were classified as the P/R subtype indicated in the top bar. A red color indicates that the signatures listed on the right is significantly enriched among the samples of that particular data set and subtype, the brighter the red the more significant, while the blue indicates the absence of a significant enrichment 
and remodeling also agrees with previous notions. Indeed, the remodeling signatures are characteristic features of mesenchyme development. However, to our knowledge, there have been no previous studies comprehensively analyzing the interrelation between ESC features and mesenchymal development in cancer. Our analysis indicates that these features might be expressions of stem-like structures at different tiers of organization.

Proliferation/remodeling subtypes across cancers of different sites. The correlation of the proliferation and remodeling signatures across human cancers indicate that they represent partially independent processes. To investigate this relationship in more detail, we investigate the occurrence of tumors with different proliferation/remodeling phenotypes. To this end, we classify each tumor sample into having $(P+)$ or not having $(P-)$ a significant upregulation of the proliferation signature and into having $(R+)$ or not having $(R-)$ a significant upregulation of the remodeling signature. Putting together the $P$ and $R$ classification, we define four possible subtypes: $\mathrm{P}-/ \mathrm{R}-, \mathrm{P}-/ \mathrm{R}+, \mathrm{P}+/ \mathrm{R}-$ and $\mathrm{P}+/ \mathrm{R}+$. In all cancers analyzed (lung, prostate, breast, colorectal, brain and ovarian), a significant number of samples were found in each of the four subtypes. This is illustrated in Figure $2 a$ for the four major cancers by incidence (lung, prostate, breast and colorectal) and in Figure $2 b$ for all cancers analyzed.

We observe a significant enrichment of gene signatures for Myc activation and p53 and PTEN inactivation in $\mathrm{P}+(\mathrm{P}+/$ $\mathrm{R}-$ and $\mathrm{P}+/ \mathrm{R}+$ ) tumors and for RAS and HIF- $1 \alpha$ activation in $\mathrm{R}+$ tumors $(\mathrm{P}-/ \mathrm{R}+$ and $\mathrm{P}+/ \mathrm{R}+)$, in agreement with the correlation analysis discussed above (Table 1$)$. The glycolysis signature is highly enriched in the $\mathrm{P}+$ subtypes in most cancers. Glycolysis is promoted by both Myc and HIF-1 $\alpha^{32}$ However, the Myc signature is enriched in the $\mathrm{P}+$ subtypes, whereas the HIF- $1 \alpha$ signature is enriched in the $R+$ subtypes. Taken together, these data indicate that the upregulation of glycolysis correlates more with Myc than HIF- $1 \alpha$ activation. The correlation analysis indicated a positive correlation between OxPhos and proliferation, but a negative correlation between OxPhos and remodeling. Now, looking at the different subtypes, we observe that OxPhos is enriched in the $\mathrm{P}+/ \mathrm{R}$ - subtype. OxPhos is activated by Myc but inhibited by HIF- $1 \alpha$. Our observations indicate that $\mathrm{HIF}-1 \alpha$ is the dominant factor regarding the activity of OxPhos, in agreement with previous work. ${ }^{33}$ The SOG pathway is enriched in the $\mathrm{P}+$ subtypes. This is consistent with the fact that most of the genes in this pathway are activated by Myc. ${ }^{23}$ We note, however, that the enrichment is stronger in the $\mathrm{P}+/ \mathrm{R}-$ than in the $\mathrm{P}+/ \mathrm{R}+$ subtype, suggesting that some signaling pathways involved in remodeling may inhibit the activity of the SOG pathway. Finally, we recapitulate the association between the ESC signature and proliferation and the association between mesenchymal development and remodeling, across most tumor sites.

Association with patient survival. To bring the proliferation/remodeling picture into a clinical context, we analyzed clinical data associated with the different cancers, focusing on overall- or disease-specific-survival (Figure 3). The current knowledge would suggest that both the $\mathrm{P}+$ and $\mathrm{R}+$ phenotypes associate with tumor aggressiveness and poor prognosis. A bird's eye inspection of the KaplanMeier plots in Figure 3 supports this expectation. Indeed, the $\mathrm{P}-/ \mathrm{R}$ - is the group with best prognosis either alone or shared with the $\mathrm{P}+/ \mathrm{R}$ - or $\mathrm{P}-/ \mathrm{R}+$ subtypes. There are, however, some cancer-specific differences regarding whether proliferation or remodeling is the major factor determining survival.

In lung, prostate, breast and brain tumors, the data sets analyzed here indicate that proliferation is the major prognostic factor. In lung tumors (Figure 3a), there is a significant splitting of the survival plots for the $\mathrm{P}+$ and $\mathrm{P}-$ subtypes $\left(P=2.7 \times 10^{-4}\right.$, log-rank test). In prostate tumors (Figure $3 b$ ), the $\mathrm{P}+/ \mathrm{R}+$ group has clearly the poorest prognosis $\left(P=3.1 \times 10^{-5}, \mathrm{P}+/ \mathrm{R}+\right.$ versus the rest), followed up by the $\mathrm{P}+/ \mathrm{R}-\left(P=1.2 \times 10^{-5}, \mathrm{P}+\right.$ versus $\left.\mathrm{P}-\right)$. In breast tumors (Figures $3 c$ and $d$ ), we observe a significant splitting of the survival plots for the $\mathrm{P}+$ and $\mathrm{P}-$ subtypes ( $P=5.6 \times 10^{-4}$ and $P=1.3 \times 10^{-7}$, respectively). In brain tumors (Figures $3 e$ and $f$ ), the $P+$ and $P-$ subtypes again result in a significant splitting $\left(P=2.9 \times 10^{-9}\right.$ and $P=5.2 \times 10^{-3}$, respectively). However, in one cohort, the survival plot for the $\mathrm{P}+/ \mathrm{R}$ - group overlaps with that for the $\mathrm{P}-/ \mathrm{R}+$ group (Figure 3e), whereas in another one, the plots for $\mathrm{P}+/ \mathrm{R}+$ and $\mathrm{P}+/ \mathrm{R}-$ groups overlap and are separated from the $P-/ R+$ plot (Figure 3f).

The pattern changes in colorectal (Figure $3 \mathrm{~g}$ ) and ovarian tumors (Figure $3 \mathrm{~h}$ ), where the data indicate that remodeling is the factor determining survival. In both cases, we see a significant splitting of the survival plots between the $\mathrm{R}+$ and $\mathrm{R}$ - subtypes $\left(P=1.0 \times 10^{-4}\right.$ in colorectal and $P=3.7 \times 10^{-4}$ in ovarian tumors, respectively). It has been previously reported that reduced proliferation associates with enhanced malignancy in colon cancer. ${ }^{34}$ Other reports state that EMT predicts poor outcome in colon cancer. ${ }^{35}$ These associations can be explained by our analysis, showing that remodeling associates with poor prognosis and proliferation correlates negatively with remodeling. To be precise, in colorectal cancers without the remodeling signature, increased proliferation associates with poor prognosis $(P+/ R-$ versus $\mathrm{P}-/ \mathrm{R}-$ ). In contrast, in colorectal cancers with the remodeling signature there is not a significant association between proliferation and survival $(\mathrm{P}+/ \mathrm{R}+$ versus $\mathrm{P}-/ \mathrm{R}+)$. The latter observation underscores the need to simultaneously consider both the proliferation and remodeling signatures to make any assessment of their impact on survival.

\section{Discussion}

The analysis and understanding of patterns of deregulation in cancer and their correlation with clinical outcome has come a long way. Underlying the enormous body of work describing individual gene malfunctions, pathway deregulation and more general oncogenic or tumor-promoting events, there is an organization of recurring themes, which concern the study of the cancer cell itself, cancer tissue and tumor formation, and systemic disease. The most prominent of these themes are proliferation and tissue remodeling. Many individual instances of these basic processes have been previously 

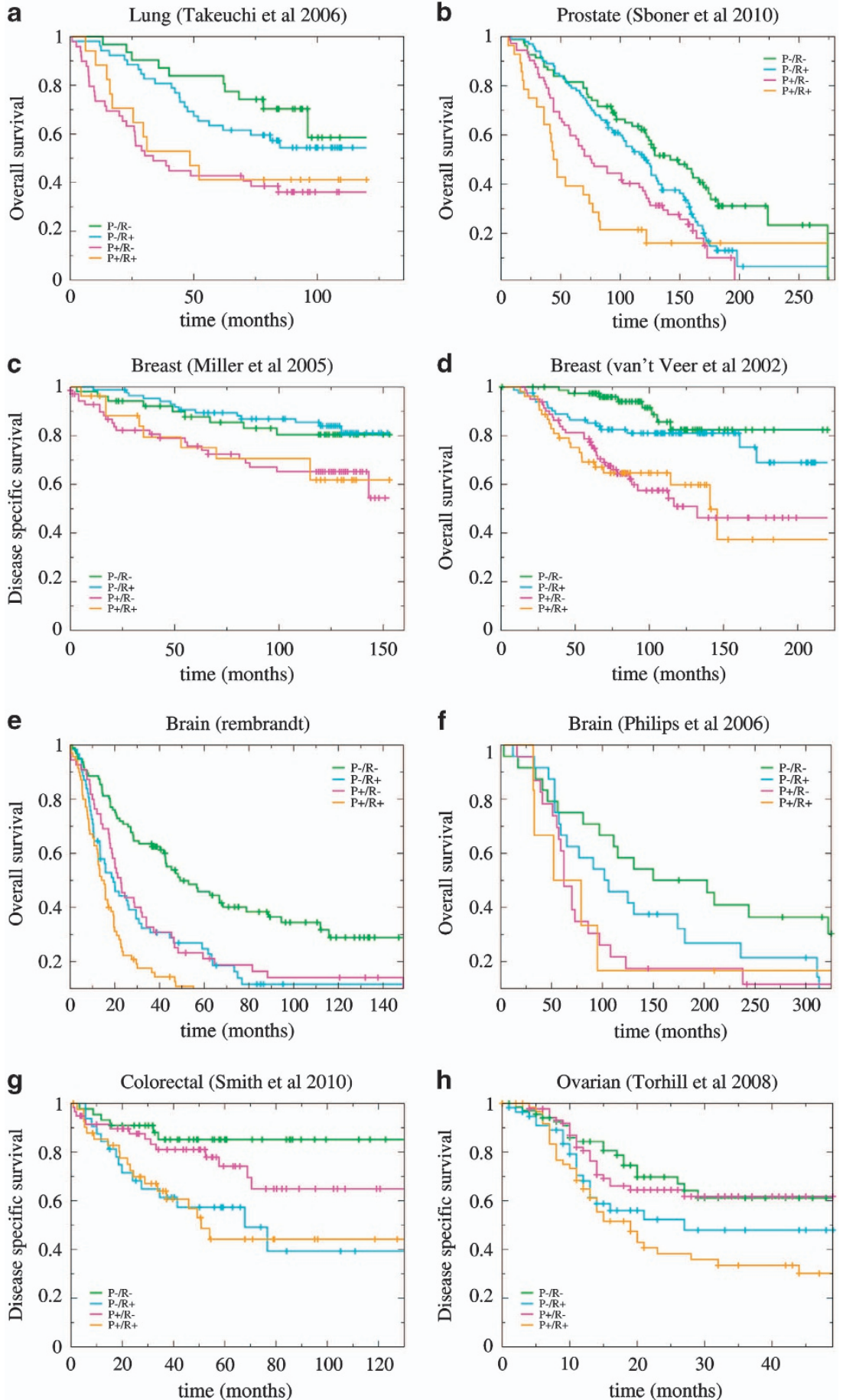

Figure 3 Proliferation-remodeling impact on survival. Kaplan-Meier survival plots stratified by the P/R subtypes in different cancers. The panels (a-h) represent different cancer types as indicated on the top of each panel.

studied and evaluated with respect to their potential to induce metastatic disease and cancer death. For instance, an association between proliferation and poor prognosis has been noticed before in the context of breast cancers, ${ }^{36,37}$ and in renal and lung cancers as well. ${ }^{38}$ Other studies have reported associations between signatures characteristic of remodeling, including hypoxia ${ }^{39}$ and mesenchymal development. 35
Our comprehensive analysis reveals that proliferation and remodeling can indeed be thought of as two distinct metapathways characterizing cancers across sites; and that these programs can have a different impact on patient survival depending on the cancer type. In most cancers tested, the $\mathrm{P}+$ groups have the worst outcome. However, we found that in colon and ovarian tissue, the $\mathrm{R}+$ groups had a significantly worse survival outcome than the $\mathrm{P}+$ groups. This might be 
explained by the high normal prolific activity within the colon (crypts) and the ovaries (follicles), as in an already highly prolific environment, structural change might have higher impact on tumor formation than additional proliferation. However, we only point this out as an interesting observation that deserves closer inspection.

In general, our observations suggest that the defining hallmarks of cancer as formulated by Hanahan-Weinberg ${ }^{1,40}$ can be organized into the meta-pathways proliferation and remodeling. Clearly, sustained proliferative signaling, evasion of growth-suppressing signals and replicative immortality are hallmarks leading to sustained proliferation. The acquisition of pluripotency or ESC phenotype, based on our analysis, goes hand in hand with these proliferative hallmarks. On the other hand, promotion of inflammation, induction of angiogenesis and activation of invasion can all be seen as an effort of the tumor to form a structure viable for growth and independent of its originally destined organ tissue. This meta-pathway also subsumes mesenchymal development or EMT phenotype, which is shown here to associate mainly with remodeling. The meta-pathways are glued together by two enabling hallmarks: 'deregulated metabolism' and 'genome instability and mutation', with which they interact. Both proliferation and remodeling represent metabolic investment strategies; as such, they will of course both require and/or depend on alterations in the metabolic regimes providing energy and building materials. The ability to satisfy these metabolic requirements in turn depends on the tumor structure and microenvironment, particularly the level of vascularization. Genome instability and mutation, as already pointed out by Hanahan and Weinberg, ${ }^{40}$ enables many of the symptomatic hallmarks associated with both proliferation and remodeling, as was documented here by the signatures for Myc, RAS, HIF- $1 \alpha$ and $\mathrm{NF}_{\kappa} \mathrm{B}$ pathway activation and $\mathrm{p} 53$ and PTEN pathway inactivation.

Following our own reasoning, we could continue to ask whether cancers manifesting the two meta-pathways together $(\mathrm{P}+/ \mathrm{R}+)$ carry some emerging properties that are not established by either of the meta-pathways independently. These could then shift the disease to the systemic level, inducing metastasis, hijacking immune response, and transforming the organism metabolism (cachexia). Our analysis indicates that the $\mathrm{P}+/ \mathrm{R}+$ group has the overall poorest prognosis. It is tempting to speculate that $\mathrm{P}+/ \mathrm{R}+$ cancers represent the most virulent state of the disease, a cancer with such strong tissue-independent organization and growth momentum, that it can disseminate itself throughout the body, inevitably leading to death. It is also possible that at the time of death, excluding inflammation and non-cancer-related deaths, all patients carry tumors in this super-cancer state.

\section{Materials and Methods}

Gene signature analysis. The following signatures were constructed based on gene ontology terms: $G_{1} / S$ transition (GO:000082), DNA replication (GO:0006260), telomere organization (GO:0032200), DNA packaging (GO:0006323), chromosome segregation (GO:0007059), $\mathrm{G}_{2} / \mathrm{M}$ transition (GO: 0000086), cell division (GO:0051301), cell junction organization (GO:0034330), cell adhesion (GO:0007155), cell migration (GO:0016477), angiogenesis (GO:0001525), cytokine production (GO:0001816), inflammatory response (GO:0006954), response to wounding (GO:0009611), glycolysis (GO:0006096), OxPhos (GO:0006119) and mesenchyme development (GO:0060485). The SOG pathway signature was constructed using the gene list reported in Vazquez et al. ${ }^{23}$ The remaining signatures were obtained from literature reports: p53 inactivation, ${ }^{41}$ PTEN inactivation, ${ }^{42}$ Myc targets, ${ }^{43} \mathrm{ESC}^{28,44}$ and HIF- $1 \alpha$ targets, ${ }^{45}$ and $\mathrm{NF} \kappa \mathrm{B}$ targets. ${ }^{46}$ The genes contained in each signature are reported in the Supplementary Table 1. For each signature, we used gene set enrichment analysis $^{47}$ to determine concordant up- or downregulation of all genes in the signature compared with the overall mean expression of genes. We use as readout heatmaps, where a red color indicates significant positive association with the signature (' $O N$ ', $P+<0.05$ ), a blue color indicates negative association with the signature ('OFF', $\mathrm{P}-<0.05$ ) and a black color indicates no significant association.

Cancer data sets. The cancer data sets analyzed here are described in Supplementary Table 2.

Clustering. Cancer samples were clustered based on their proliferation and remodeling signature. A sample was defined $\mathrm{P}+$ whenever it manifested a significant expression of the proliferation signature, $\mathrm{P}$ - otherwise. A sample was defined $\mathrm{R}+$ whenever it manifested a significant expression of the remodeling meta-signature, $\mathrm{R}$ - otherwise. The samples were then clustered into the four possible subtypes $\mathrm{P}-/ \mathrm{R}-, \mathrm{P}-/ \mathrm{R}+, \mathrm{P}+/ \mathrm{R}-$ and $\mathrm{P}+/ \mathrm{R}+$.

\section{Conflict of Interest}

The authors declare no conflict of interest.

Acknowledgements. This research was supported by NCI P30 CA072720 and $\mathrm{NCl}$ P01 CA087497-11.

1. Hanahan D, Weinberg RA. The hallmarks of cancer. Cell 2000; 100: 57-70.

2. Fritz V, Fajas L. Metabolism and proliferation share common regulatory pathways in cancer cells. Oncogene 29: 4369-4377.

3. Vogelstein B, Kinzler KW. Cancer genes and the pathways they control. Nat Med 2004; 10 : 789-799.

4. Kerbel RS. Tumor angiogenesis. N Engl J Med 2008; 358: 2039-2049.

5. Grivennikov SI, Greten FR, Karin M. Immunity, inflammation, and cancer. Cell 2010; 140 : 883-899.

6. Keith B, Simon MC. Hypoxia-inducible factors, stem cells, and cancer. Cell 2007; 129 : 465-472.

7. Leber MF, Efferth T. Molecular principles of cancer invasion and metastasis (review). Int J Oncol 2009; 34: 881-895.

8. Blum D, Omlin A, Baracos VE, Solheim TS, Tan BH, Stone P et al. Cancer cachexia: a systematic literature review of items and domains associated with involuntary weight loss in cancer. Crit Rev Oncol Hematol 80: 114-144.

9. Sjoblom T, Jones S, Wood LD, Parsons DW, Lin J, Barber TD et al. The consensus coding sequences of human breast and colorectal cancers. Science (80- ) 2006; 314: 268-274.

10. Jones S, Zhang X, Parsons DW, Lin JC, Leary RJ, Angenendt $P$ et al. Core signaling pathways in human pancreatic cancers revealed by global genomic analyses. Science 2008; 321: 1801-1806.

11. Parsons DW, Li M, Zhang X, Jones S, Leary RJ, Lin JC et al. The genetic landscape of the childhood cancer medulloblastoma. Science 2011; 331: 435-439.

12. Ben-Porath I, Thomson MW, Carey VJ, Ge R, Bell GW, Regev A et al. An embryonic stem cell-like gene expression signature in poorly differentiated aggressive human tumors. Nat Genet 2008; 40: 499-507.

13. Mizuno H, Spike BT, Wahl GM, Levine AJ. Inactivation of p53 in breast cancers correlates with stem cell transcriptional signatures. Proc Natl Acad Sci USA 107: 22745-22750.

14. Markert EK, Mizuno H, Vazquez A, Levine AJ. Molecular classification of prostate cancer using curated expression signatures. Proc Natl Acad Sci USA. 2011; 108: 21276-21281.

15. Mani SA, Guo W, Liao MJ, Eaton EN, Ayyanan A, Zhou AY et al. The epithelialmesenchymal transition generates cells with properties of stem cells. Cell 2008; 133: 704-715.

16. Battula VL, Evans KW, Hollier BG, Shi Y, Marini FC, Ayyanan A et al. Epithelialmesenchymal transition-derived cells exhibit multilineage differentiation potential similar to mesenchymal stem cells. Stem Cells 28: 1435-1445.

17. Iwatsuki M, Mimori K, Yokobori T, Ishi H, Beppu T, Nakamori S et al. Epithelialmesenchymal transition in cancer development and its clinical significance. Cancer Sci 101: 293-299.

18. Pylayeva-Gupta $Y$, Grabocka E, Bar-Sagi D. RAS oncogenes: weaving a tumorigenic web. Nat Rev Cancer 2011; 11: 761-774.

19. Ying H, Kimmelman AC, Lyssiotis CA, Hua S, Chu GC, Fletcher-Sananikone E et al. Oncogenic Kras Maintains Pancreatic Tumors through Regulation of Anabolic Glucose Metabolism. Cell 2012; 149: 656-670. 
20. Hart GW, Copeland RJ. Glycomics hits the big time. Cell 2010; 143: 672-676.

21. Locasale JW, Cantley LC. Metabolic flux and the regulation of mammalian cell growth. Cell Metab 2011; 14: 443-451.

22. Lemons JM, Feng XJ, Bennett BD, Legesse-Miller A, Johnson EL, Raitman I et al. Quiescent fibroblasts exhibit high metabolic activity. PLOS Biol 2010; 8: e1000514.

23. Vazquez A, Markert EK, Oltvai ZN. Serine biosynthesis with one carbon catabolism and the glycine cleavage system represents a novel pathway for ATP generation. PLoS One 2011; 6: e25881.

24. Possemato R, Marks KM, Shaul YD, Pacold ME, Kim D, Birsoy K et al. Functional genomics reveal that the serine synthesis pathway is essential in breast cancer. Nature 2011; 476: 346-350.

25. Pollari S, Kakonen SM, Edgren $\mathrm{H}$, Wolf M, Kohonen $\mathrm{P}$, Sara $\mathrm{H}$ et al. Enhanced serine production by bone metastatic breast cancer cells stimulates osteoclastogenesis. Breast Cancer Res Treat 2011; 125: 421-430.

26. Locasale JW, Grassian AR, Melman T, Lyssiotis CA, Mattaini KR, Bass AJ et al. Phosphoglycerate dehydrogenase diverts glycolytic flux and contributes to oncogenesis. Nat Genet 2011; 43: 869-874.

27. Morel AP, Lievre M, Thomas C, Hinkal G, Ansieau S, Puisieux A. Generation of breast cancer stem cells through epithelial-mesenchymal transition. PLos One 2008; 3: e2888.

28. Mizuno H, Spike BT, Wahl GM, Levine AJ. Inactivation of p53 in breast cancers correlates with stem cell transcriptional signatures. Proc Natl Acad Sci USA 2010; 107: 22745-22750.

29. Woo HG, Wang XW, Budhu A, Kim YH, Kwon SM, Tang ZY et al. Association of TP53 mutations with stem cell-like gene expression and survival of patients with hepatocellular carcinoma. Gastroenterology 2011; 140: 1063-1070.

30. Amit M, Carpenter MK, Inokuma MS, Chiu CP, Harris CP, Waknitz MA et al. Clonally derived human embryonic stem cell lines maintain pluripotency and proliferative potential for prolonged periods of culture. Dev Biol 2000; 227: 271-278.

31. Stojkovic M, Lako M, Strachan T, Murdoch A. Derivation, growth and applications of human embryonic stem cells. Reproduction 2004; 128: 259-267.

32. Dang CV, Kim JW, Gao P, Yustein J. The interplay between MYC and HIF in cancer. Nat Rev Cancer 2008; 8: 51-56.

33. Papandreou I, Cairns RA, Fontana L, Lim AL, Denko NC. HIF-1 mediates adaptation to hypoxia by actively downregulating mitochondrial oxygen consumption. Cell Metab 2006; 3: $187-197$.

34. Anjomshoaa A, Lin YH, Black MA, McCall JL, Humar B, Song S et al. Reduced expression of a gene proliferation signature is associated with enhanced malignancy in colon cancer. Br J Cancer 2008; 99: 966-973.

35. Loboda A, Nebozhyn MV, Watters JW, Buser CA, Shaw PM, Huang PS et al. EMT is the dominant program in human colon cancer. BMC Med Genomics 2011; 4: 9.

36. Chang HY, Nuyten DS, Sneddon JB, Hastie T, Tibshirani R, Sorlie T et al. Robustness, scalability, and integration of a wound-response gene expression signature in predicting breast cancer survival. Proc Natl Acad Sci USA. 2005; 102: 3738-3743.

37. Whitfield ML, George LK, Grant GD, Perou CM. Common markers of proliferation. Nat Rev Cancer 2006; 6: 99-106.

38. Starmans MH, Krishnapuram B, Steck $\mathrm{H}$, Horlings $\mathrm{H}$, Nuyten DS, van de Vijver MJ et al. Robust prognostic value of a knowledge-based proliferation signature across large patient microarray studies spanning different cancer types. Br J Cancer 2008; 99: 1884-1890.

39. Chi JT, Wang Z, Nuyten DS, Rodriguez EH, Schaner ME, Salim A et al. Gene expression programs in response to hypoxia: cell type specificity and prognostic significance in human cancers. PLoS Med 2006; 3: e47.

40. Hanahan D, Weinberg RA. Hallmarks of cancer: the next generation. Cell 2011; 144 646-674.

41. Miller LD, Smeds J, George J, Vega VB, Vergara L, Ploner A et al. An expression signature for p53 status in human breast cancer predicts mutation status, transcriptional effects, and patient survival. Proc Natl Acad Sci USA. 2005; 102: 13550-13555.

42. Saal LH, Johansson P, Holm K, Gruvberger-Saal SK, She QB, Maurer M et al. Poor prognosis in carcinoma is associated with a gene expression signature of aberrant PTEN tumor suppressor pathway activity. Proc Natl Acad Sci USA 2007; 104: 7564-7569.

43. Zeller KI, Jegga AG, Aronow BJ, O'Donnell KA, Dang CV. An integrated database of genes responsive to the Myc oncogenic transcription factor: identification of direct genomic targets. Genome Biol 2003; 4: R69.

44. Assou S, Le Carrour T, Tondeur S, Strom S, Gabelle A, Marty S et al. A meta-analysis of human embryonic stem cells transcriptome integrated into a web-based expression atlas. Stem Cells 2007; 25: 961-973.

45. Benita $Y$, Kikuchi $H$, Smith AD, Zhang MQ, Chung DC, Xavier RJ. An integrative genomics approach identifies Hypoxia Inducible Factor-1 (HIF-1)-target genes that form the core response to hypoxia. Nucleic Acids Res 2009; 37: 4587-4602.

46. Schreiber J, Jenner RG, Murray HL, Gerber GK, Gifford DK, Young RA. Coordinated binding of NF-kappaB family members in the response of human cells to lipopolysaccharide. Proc Natl Acad Sci USA. 2006; 103: 5899-5904.

47. Subramanian A, Tamayo P, Mootha VK, Mukherjee S, Ebert BL, Gillette MA et al. Gene set enrichment analysis: a knowledge-based approach for interpreting genome-wide expression profiles. Proc Natl Acad Sci USA. 2005; 102: 15545-15550.

48. Creighton CJ, Casa A, Lazard Z, Huang S, Tsimelzon A, Hilsenbeck SG et al. Insulin-like growth factor-I activates gene transcription programs strongly associated with poor breast cancer prognosis. J Clin Oncol 2008; 26: 4078-4085.

49. Sartor MA, Mahavisno V, Keshamouni VG, Cavalcoli J, Wright Z, Karnovsky A et al. ConceptGen: a gene set enrichment and gene set relation mapping tool. Bioinformatics 2010; 26: 456-463.

(c)

SOMERIAHISRESERV

Cell Death and Disease is an open-access journal published by Nature Publishing Group. This work is licensed under the Creative Commons Attribution-NonCommercial-No Derivative Works 3.0 Unported License. To view a copy of this license, visit http://creativecommons.org/licenses/by-nc-nd/3.0/

Supplementary Information accompanies the paper on Cell Death and Disease website (http://www.nature.com/cddis) 